OPEN ACCESS

Edited by:

Giuseppe Losurdo,

University of Bari Medical School, Italy

Reviewed by:

Yuji Naito,

Kyoto Prefectural University of

Medicine, Japan

Enzo leardi,

University of Bari Aldo Moro, Italy

Chafia Boukoffa Touil-Boukoffa,

University of Science and Technology

Houari Boumediene, Algeria

*Correspondence:

Bing Chang

cb000216@163.com

Specialty section:

This article was submitted to

Gastroenterology,

a section of the journal

Frontiers in Medicine

Received: 29 May 2021 Accepted: 05 August 2021

Published: 31 August 2021

Citation:

Wu Z, Pan D, Jiang $M$, Sang $L$ and Chang B (2021) Selenium-Enriched

Lactobacillus acidophilus Ameliorates

Dextran Sulfate Sodium-Induced Chronic Colitis in Mice by Regulating Inflammatory Cytokines and Intestinal

Microbiota. Front. Med. 8:716816.

doi: 10.3389/fmed.2021.716816

\section{Selenium-Enriched Lactobacillus acidophilus Ameliorates Dextran Sulfate Sodium-Induced Chronic Colitis in Mice by Regulating Inflammatory Cytokines and Intestinal Microbiota}

\author{
Zeyu Wu ${ }^{1}$, Dan Pan ${ }^{2}$, Min Jiang ${ }^{1}$, Lixuan Sang ${ }^{3}$ and Bing Chang ${ }^{1 *}$ \\ ${ }^{1}$ Department of Gastroenterology, First Affiliated Hospital of China Medical University, Shenyang, China, ${ }^{2}$ Department of \\ Geriatrics, First Affiliated Hospital of China Medical University, Shenyang, China, ${ }^{3}$ Department of Gastroenterology, Shengjing \\ Hospital of China Medical University, Shenyang, China
}

Aim: To evaluate the effect of Selenium-enriched Lactobacillus acidophilus (Se-enriched L. acidophilus) on dextran sulfate sodium (DSS)-induced colitis in mice.

Methods: Mice were randomly divided into four groups: a control group, a control + Se-enriched L. acidophilus group, a chronic colitis group, and a chronic colitis + Seenriched $L$. acidophilus group ( $n=10$ each group). The mice were sacrificed on the 26th day. The disease activity index, survival rates, and histological injury score were determined. Cytokines produced by lamina propria lymphocytes (LPLs), the selenium (Se) concentrations in serum and colon tissue and the mouse intestinal microbiota were evaluated.

Results: Se-enriched $L$. acidophilus can improve histological injury and the disease activity index in mice with chronic colitis and reduce $\mathrm{IL}-1 \beta, \mathrm{IL}-6, \mathrm{IL}-12 \mathrm{p} 70, \mathrm{TNF}-\alpha$, IL-23, IFN- $\gamma$, IL-17A, and IL-21 $(P<0.05)$ and increase IL-10 $(P<0.05)$ expression levels. Moreover, Se-enriched $L$. acidophilus can increase the $\beta$ diversity of intestinal microbiota in mice with chronic colitis, significantly reduce the relative abundance of Lactobacillus and Romboutsia $(P<0.05)$, and significantly increase the relative abundance of Parasutterella $(P<0.05)$.

Conclusions: Se-enriched L. acidophilus can improve DSS-induced chronic colitis by regulating inflammatory cytokines and intestinal microbiota.

\footnotetext{
Keywords: inflammatory bowel disease, ulcerative colitis, Se-enriched Lactobacillus acidophilus, intestinal flora, molecular pathological epidemiology
}

\section{INTRODUCTION}

Inflammatory bowel disease (IBD) is a chronic recurrent inflammatory disease of the intestine that mainly includes two forms, ulcerative colitis (UC) and Crohn's disease (CD) (1), and its prevalence is increasing annually (2). The pathogenesis of IBD is not fully understood. Genes, immunity, intestinal flora, and the environment are all involved in the pathogenesis of IBD (3). There are a 
large number of microbiota in the human intestine, which has an important impact on the human body, and disorders of the intestinal flora are considered to be closely related to the occurrence and development of IBD (4). Studies have shown that the treatment methods for regulating the intestinal flora such as fecal bacteria transplantation (FMT) (5-7), and VSL\#3 probiotic treatment $(8,9)$ could be used in the treatment of ulcerative colitis.

Lactobacillus acidophilus (L. acidophilus) is an important probiotic (10) that has a certain therapeutic effect on many diseases. L. acidophilus can alleviate the pain caused by osteoarthritis and delay the progression of osteoarthritis by reducing the destruction of cartilage and inhibiting the production of proinflammatory cytokines (11). It also has a certain relieving effect on type 2 diabetes (12). Obesity and fatty liver caused by diet can also be relieved by L. acidophilus through improving fat metabolism and insulin sensitivity (13). L. acidophilus can also inhibit endoplasmic reticulum stress (ER), thereby alleviating intestinal inflammation (14). In addition, evodiamine can relieve dextran sulfate sodium (DSS)-induced colitis by increasing L. acidophilus in the intestine (15).

Selenium (Se) is an important trace element in the human body that has antioxidant and anti-inflammatory effects and has an important influence on human immunity $(16,17)$. Clinical studies have found that compared with healthy people, CD patients exhibit significantly reduced concentrations of selenoprotein $\mathrm{P}$ and Se $(18,19)$, and the concentration of Se in UC patients is also significantly reduced (20). Moreover, it was reported that sodium selenite can alleviate DSS-induced colitis in mice (21).

The preparation of Se-enriched probiotics adopts the biological transformation method, in which inorganic Se is added during the probiotic culture process, and the probiotics take up inorganic Se and convert it into organic Se, which is then transformed into Se-enriched probiotics (22). Studies have found that Se-enriched probiotics can reduce liver damage induced by carbon tetrachloride $(23,24)$. The anti-inflammatory and antioxidant effects of Se-enriched probiotics can also improve the liver damage induced by heat stress in rats (25).

Since both Se and probiotics alleviate intestinal inflammation, Se-enriched probiotics may alleviate intestinal inflammation. Therefore, our study established a DSS-induced mouse colitis model to study the effect of Se-enriched L. acidophilus on intestinal inflammation and its possible mechanism.

\section{EXPERIMENTAL METHOD}

\section{Experimental Animals and Probiotics}

Forty 8-week-old specific pathogen-free C57BL/6 male mice were purchased from Liaoning Changsheng Biology, each weighing $22 \pm 2 \mathrm{~g}$ and bred under specific pathogen-free conditions (temperature $21-25^{\circ} \mathrm{C}$, humidity $50-60 \%$, and a $12 \mathrm{~h}$ light $/ 12 \mathrm{~h}$ dark-light regimen). Se-enriched L. acidophilus is a freeze-dried powder produced by the Immunology Laboratory of China Medical University. Each gram of freeze-dried powder contains Se-enriched L. acidophilus $5 \times 10 \sim 9 \mathrm{cfu}$, and the selenium content is $0.30 \mathrm{mg} / \mathrm{g}$. The research protocol was approved by the
Animal Ethics Committee and Animal Care Committee of China Medical University. Ethics batch number: 2019069.

\section{Experimental Design}

Forty mice were randomly divided into four groups: 10 in the control group (group A), 10 in the control + Se-enriched $L$. acidophilus group (group B), 10 in the chronic colitis group (group C), and 10 in the chronic colitis + Se-enriched $L$. acidophilus group (group D). The control group was given a normal diet and tap water, with normal saline gavage once a day. The control + Se-enriched $L$. acidophilus group was given a normal diet and tap water, with Se-enriched L. acidophilus (100 $\mathrm{mg} / \mathrm{kg}$ ) gavage once a day. The chronic colitis group was induced colitis by $1.5 \%$ DSS and given a normal diet with saline gavage once a day. The chronic colitis + Se-enriched L. acidophilus group was induced colitis by $1.5 \%$ DSS and given a normal diet with Se-enriched L. acidophilus (100 $\mathrm{mg} / \mathrm{kg})$ gavage once a day. Weight and disease activity index were recorded every day.

\section{Induction of Chronic DSS Colitis}

Colitis was induced in the mice by oral administration of $1.5 \%$ DSS (molecular mass 36-50 kDa; MP Biomedicals, Solon, $\mathrm{OH}$, United States) on days 0-5, 10-15, and 20-25 d and tap water on the other days (26). The mice were sacrificed on 26 th day.

\section{Disease Activity Index}

The disease activity index was used to assess the severity of colitis in mice. It consists of three parts, the percentage of weight loss (0-4 points), stool consistency (0-4 points), and intestinal bleeding (0-4 points) (26), as shown in Table 1. After the mice were sacrificed, the colon tissue was fixed with $4 \%$ paraformaldehyde, embedded in paraffin, cut into $4-\mu \mathrm{m}$ sections, stained with hematoxylin and eosin, and scored for histological damage. Histological scores were assessed by two pathologists independently in a blinded fashion. The histological scores were obtained by calculating the sum of scores of inflammation severity, degree of mucosal damage, percentage of crypt damage, and pathological change range. The none, mild, moderate, or severe inflammation was quantified as to the percentage involvement by the inflammation (none, 0-33\%, 33-67\%, 67$100 \%$ ). Depth of inflammation (none, mucous layer, submucosa, muscularis, and serosa) represented the mucosal damage, as shown in Table 2 (26).

\section{Cell Preparation, Culture, and Activation}

The large intestine of each mouse was cut into 1- to 2-mm small pieces. The pieces were stirred twice in PBS containing $3 \mathrm{mmol} / \mathrm{L}$ EDTA for $15 \mathrm{~min}$ each and twice in RPMI 1640 (HyClone) containing $1 \mathrm{mmol} / \mathrm{L}$ EGTA for $20 \mathrm{~min}$ each to remove epithelium at $37^{\circ} \mathrm{C}$. The remaining pieces were stirred in RPMI 1640 (HyClone) containing 20\% fetal bovine serum, 100 U/ml collagenase (C2139; Sigma-Aldrich Corp., St. Louis, $\mathrm{MO}$, United States) and $5 \mathrm{U} / \mathrm{ml}$ DNase1 (Sigma-Aldrich Corp) at $37^{\circ} \mathrm{C}$ for $90 \mathrm{~min}$. The suspensions were centrifuged, and the particles were cleaned. Lamina propria lymphocytes (LPLs) were isolated from lamina propria (LP) cell preparations by $45-66.6 \%$ 
TABLE 1 | Disease activity index (DAI) score chart.

\begin{tabular}{|c|c|c|c|}
\hline Score & Weight loss (\%) & Stool property & Bleeding \\
\hline 0 & 0 & Normal & Normal \\
\hline 1 & $>0-5$ & & \\
\hline 2 & $>5-10$ & Loose & Fecal occult blood \\
\hline 3 & $>10-15$ & & \\
\hline 4 & $>15$ & Diarrhea & Bleeding \\
\hline
\end{tabular}

TABLE 2 | Histological injury score chart.

\begin{tabular}{|c|c|c|c|c|c|}
\hline Grade & 0 & 1 & 2 & 3 & 4 \\
\hline Inflammation & None & Mild & Moderate & Severe & - \\
\hline Mucosal damage & None & Mucous layer & Submucosa & Muscularis and serosa & - \\
\hline Crypt damage & None & $1 / 3$ & $2 / 3$ & $100 \%$ & $100 \%$ with epithelium loss \\
\hline Pathological change range & None & $0-25 \%$ & $26-50 \%$ & $51-75 \%$ & $76-100 \%$ \\
\hline
\end{tabular}

discontinuous Percoll (Solarbio) gradient centrifugation at 2,500 rpm for $20 \mathrm{~min}$ (26).

In an atmosphere containing 5\% CO2, LPLs $\left(1 \times 10^{5} /\right.$ well in $0.2 \mathrm{ml}$ of RPMI 1640 medium containing $10 \%$ fetal bovine serum, $1 \%$ penicillin, and $1 \%$ streptomycin) were cultured in 96-well plates coated with anti-CD3 $(10 \mu \mathrm{g} / \mathrm{ml}$ e-Bioscience, San Diego, CA, United States) and soluble anti-CD28 $(1 \mu \mathrm{g} / \mathrm{ml}$, eBioscience) $\mathrm{mAbs}$ for $48 \mathrm{~h}$ at $37^{\circ} \mathrm{C}$. After $48 \mathrm{~h}$, the supernatants were collected, and the cytokine concentrations were determined by enzyme-linked immunosorbent assay (26).

\section{Enzyme-Linked Immunosorbent Assay}

According to the manufacturer's instructions, cell culture supernatants were collected after centrifugation at 1,000 rpm for $10 \mathrm{~min}$, and cytokine concentrations were measured using mouse immunoassay kits (R\&D Systems Inc., Minneapolis, MN, United States). The levels of TNF- $\alpha$, IL-1 $\beta$, IL-6, IL-23, and IL-12p70 were measured in the supernatants without antiCD3/anti-CD28 monoclonal antibody stimulation. The levels of IFN- $\gamma$, IL-17A, IL-22, IL-21, and IL-10 were measured in the supernatants with or without anti-CD28/anti-CD3 monoclonal antibody stimulation (26).

\section{Determination of Selenium in Serum and Colon Tissue}

The selenium content in colon tissue was determined by fluorescence atomic absorption spectrometry. Serum selenium concentrations were detected in duplicate by inductively coupled plasma mass spectrometry (ICP-MS, Perkin-Elmer SCIEX ElAN 6000, US) (21).

\section{DNA Extraction and Amplification}

The fecal samples of mice were transported to laboratory within $2 \mathrm{~h}$ with an ice pack. All samples were frozen immediately then and stored at $-80^{\circ} \mathrm{C}$. Realbio Genomics Institute (Shanghai, China) performed DNA extraction and amplification. The microbial DNA of the samples was extracted by a QIAamp
FAST DNA Stool Mini Kit (Item No. 51604, Qiagen, Germany) according to the instructions. The integrity and concentration of total DNA were quality tested by a Thermo NanoDrop 2000 UV spectrophotometer and 1\% agarose gel electrophoresis. Primers 341F $5^{\prime}$-CCTACGGGRSGCAGCAG- $3^{\prime}$ and 806R $5^{\prime}$ GGACTACVVGGGTATCTAATC-3' (with a specific barcode in the primer) were used to amplify the $\mathrm{V} 3-\mathrm{V} 4$ region of the bacterial $16 \mathrm{~s}$ ribosomal RNA gene by $\operatorname{PCR}\left(95^{\circ} \mathrm{C}\right.$ for $3 \mathrm{~min}$, followed by 30 cycles at $98^{\circ} \mathrm{C}$ for $20 \mathrm{~s}, 58^{\circ} \mathrm{C}$ for $15 \mathrm{~s}$, and $72^{\circ} \mathrm{C}$ for $20 \mathrm{~s}$ and a final extension at $72^{\circ} \mathrm{C}$ for $5 \mathrm{~min}$ ), and amplified fragments of approximately $500 \mathrm{bp}$ were obtained.

\section{DNA Sequencing and Analysis}

Realbio Genomics Institute (Shanghai,China) performed DNA sequencing and analysis. According to the manufacturer's instructions, the PCR products were extracted from 2\% agarose gels and purified using the AxyPrep DNA Gel Extraction Kit (Axygen Biosciences, Union City, CA, U.S.). Amplicons were quantified using Qubit 2.0 (Invitrogen, U.S.). All quantified amplicons were pooled to equalize concentrations in order to sequence using Illumina HiSeq/MiSeq (Illumina, Inc., CA, USA) and PANDAseq (https://github.com/neufeld/ pandaseq, version 2.9) was used to overlap the paired end reads of $250 \mathrm{bp}$ on their 3 ends for concatenation into original longer tags.

OTUs were clustered according to $97 \%$ similarity using UPARSE (http://drive5.com/uparse/), and USEARCH (version 7.0.1090) was used to identify and remove chimeric sequences. Each representative sequence was annotated by RDP Classifier (http://rdp.cme.msu.edu/) based on RDP Database. The OTU profiling table and alpha diversity indices (including Chaol index, Shannon index, Simpson index, observed species index, and PD-whole-tree diversity index) were achieved by the Python scripts of QIIME (version 1.9.1). Principal coordinate analysis (PCoA) based on weighted UniFrac distance and the Adonis test were implemented by R software (version 3.5.1). The microbiota differences between different groups were analyzed with linear discriminant analysis effect size (LEfSe) analysis software. The 
A

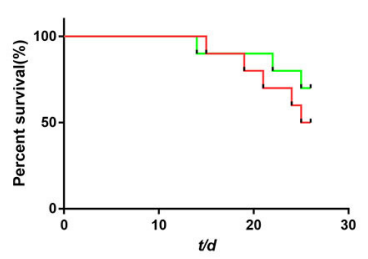

B

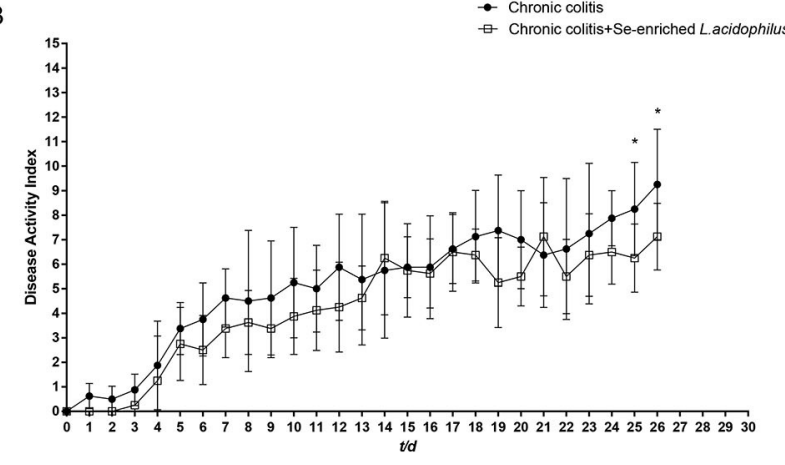

+ Chronic colitis
+ Chronic colitis+Se-enriched L.acidophilus
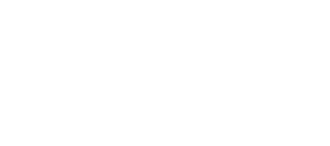

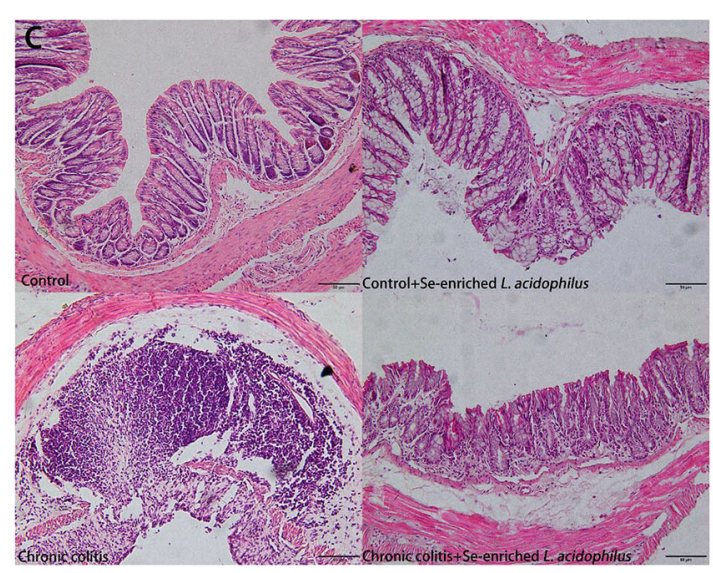

D

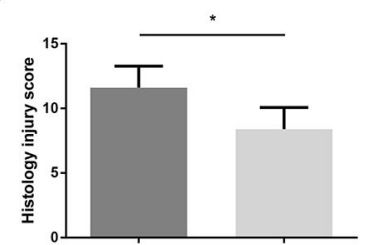

Chronic colitistSe-enriched L.acidophilus
Chronic colitis

FIGURE 1 | (A) Survival rates between the chronic colitis group and the chronic colitis + Se-enriched $L$. acidophilus group ( $n=10$ ); (B) Chronic colitis group and chronic colitis + Se-enriched L. acidophilus group DAl scores $(n=8)$; (C) H\&E staining of colon tissue of four groups. (200x) (Control) There were no inflammatory cells infiltration. (Control+Se-enriched $L$. acidophilus) There were no inflammatory cells infiltration. (Chronic colitis) Numerous neutrophil and mononuclear cells infiltration could be found. (Chronic colitis + Se-enriched $L$. acidophilus) There were fewer neutrophil and mononuclear cells infiltration than chronic colitis group. (D) Histological injury scores between the chronic colitis group and the chronic colitis + Se-enriched $L$. acidophilus group ( $n=5$ ). Data are expressed as the mean \pm standard error $\left({ }^{\star} P<0.05\right)$.

correlations between microbiota and cytokines were analyzed by R software (version 3.5.1).

\section{Data Analysis}

The data are expressed as the mean \pm standard error, and the Shapiro Wilk test was used for normality analysis. If the data conformed to a normal distribution and homogeneity of variance, analysis of variance or $t$-test was used. If the data conformed to a normal distribution and uneven variance, the Welch test or $t$ ' test was used. If the data did not conform to a normal distribution, a non-parametric test was used. $P<0.05$ indicated that the difference was statistically significant. SPSS version 22.0 (SPSS, Inc., Chicago, IL, United States) was used for data analysis, and GraphPad Prism 6.0 (GraphPad Software, Inc., La Jolla, CA, United States) was used for drawing.

\section{RESULTS}

\section{Se-Enriched L. acidophilus Improves DSS Colitis}

The effect of Se-enriched L. acidophilus on DSS colitis was compared by the differences in survival rate, DAI score and colon histology between the two groups. The chronic colitis group and the chronic colitis + Se-enriched L. acidophilus group had similar survival rates $(P>0.05)$. The DAI score of the chronic colitis + Se-enriched L. acidophilus group decreased significantly on the 25th and 26th days $(P<0.05)$, and the histological injury score of the chronic colitis + Se-enriched $L$. acidophilus group significantly decreased $(P<0.05)$, as shown in Figure 1.

\section{Se-Enriched $L$. acidophilus Regulates Cytokines}

Detection of the cytokine concentrations in the supernatant (five samples per group) revealed that IL-1 $\beta$, IL-6, IL-12p70, TNF$\alpha$, and IL-23 were significantly decreased in the chronic colitis + Se-enriched $L$. acidophilus group compared with the chronic colitis group $(P<0.05)$. Regardless of whether there was antiCD3/CD28 antibody stimulation, IFN- $\gamma$, IL-17A, and IL-21 were significantly decreased $(P<0.05)$, and IL-10 was significantly increased in the chronic colitis + Se-enriched L. acidophilus group compared with the chronic colitis group $(P<0.05)$. Only the concentration of IL-22 between the two groups was not statistically significant. Compared with the chronic colitis group, serum and colon tissue Se concentrations were significantly higher in the chronic colitis + Se-enriched L. acidophilus group $(P<0.01)$, as shown in Figure 2.

\section{Se-Enriched $L$. acidophilus Regulates Intestinal Microbiota}

Se-enriched L. acidophilus has regulatory effects on the intestinal microbiota. The feces of mice in the control, control $+\mathrm{Se}-$ enriched L. acidophilus, chronic colitis, and chronic colitis $+\mathrm{Se}-$ enriched L. acidophilus groups were collected (five samples per group). There were no significant differences in the $\alpha$ diversity 


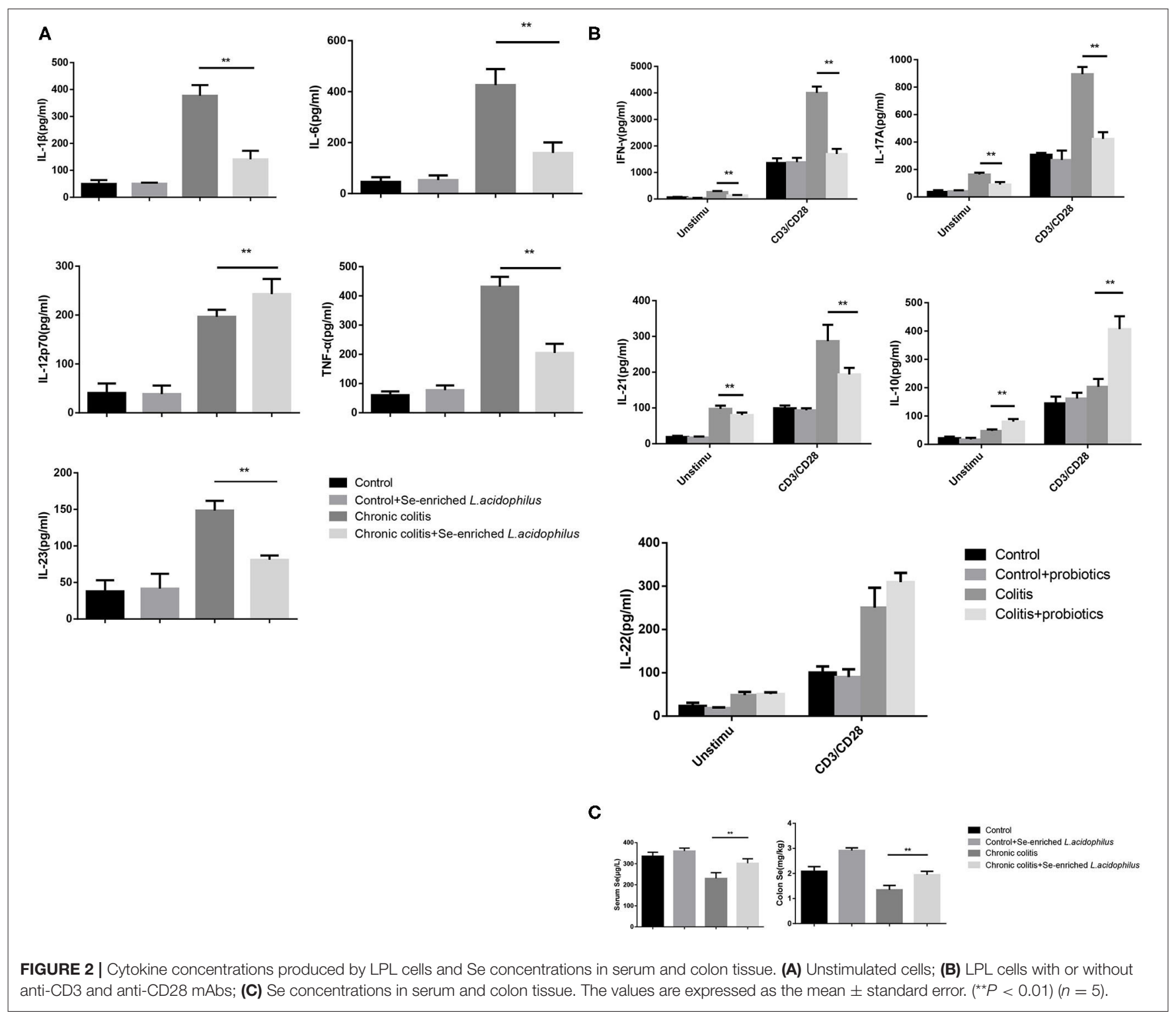

among the four groups, Chao1 index $(P>0.05)$, Shannon index $(P>0.05)$, Simpson index $(P>0.05)$, observed species index $(P$ $>0.05)$, or PD_whole_tree diversity index $(P>0.05)$, as shown in Figure 3. There was a significant difference in microbial $\beta$ diversity between the control group and the chronic colitis group $(P<0.05)$. There was also a significant difference in microbial $\beta$ diversity between the chronic colitis group and the chronic colitis + Se-enriched $L$. acidophilus group $(P<0.05)$, as shown in Figure 4.

To identify the differences in microbiota between different groups, we conducted LEfSe analysis of the dominant flora between different groups. There were differences in the composition of intestinal microbiota between the control group and the chronic colitis group and between the chronic colitis group and the chronic colitis + Se-enriched L. acidophilus group, as shown in Figure 5. At the phylum level, the control group was rich in Bacteroidetes $(P<0.05)$, and the chronic colitis group was rich in Firmicutes and Tenericutes $(P<0.05)$, as shown in Figure 6. At the genus level, compared with the chronic colitis group, the control group was rich in Helicobacter, Rikenella, Barnesiella, and Enterorhabdus, while Turicibacter, Romboutsia, Escherichia_Shigella, Clostridium sensu stricto, Butyricimonas, Parasutterella, Bifidobacterium, Allobaculum, Clostridium IV, Anaeroplasma, Intestinimonas, and Clostridium XVIII were significantly reduced $(P<0.05)$. The relative abundances of Lactobacillus and Romboutsia in the chronic colitis + Seenriched L. acidophilus group significantly decreased $(P<$ 0.05 ), and the relative abundance of Parasutterella significantly increased $(P<0.05)$, as shown in Figure 6. The relative abundance of Akkermansia increased, although the difference was not statistically significant [LDA score $(\log 10)<2$ ], as shown in Figures 5, 7. 

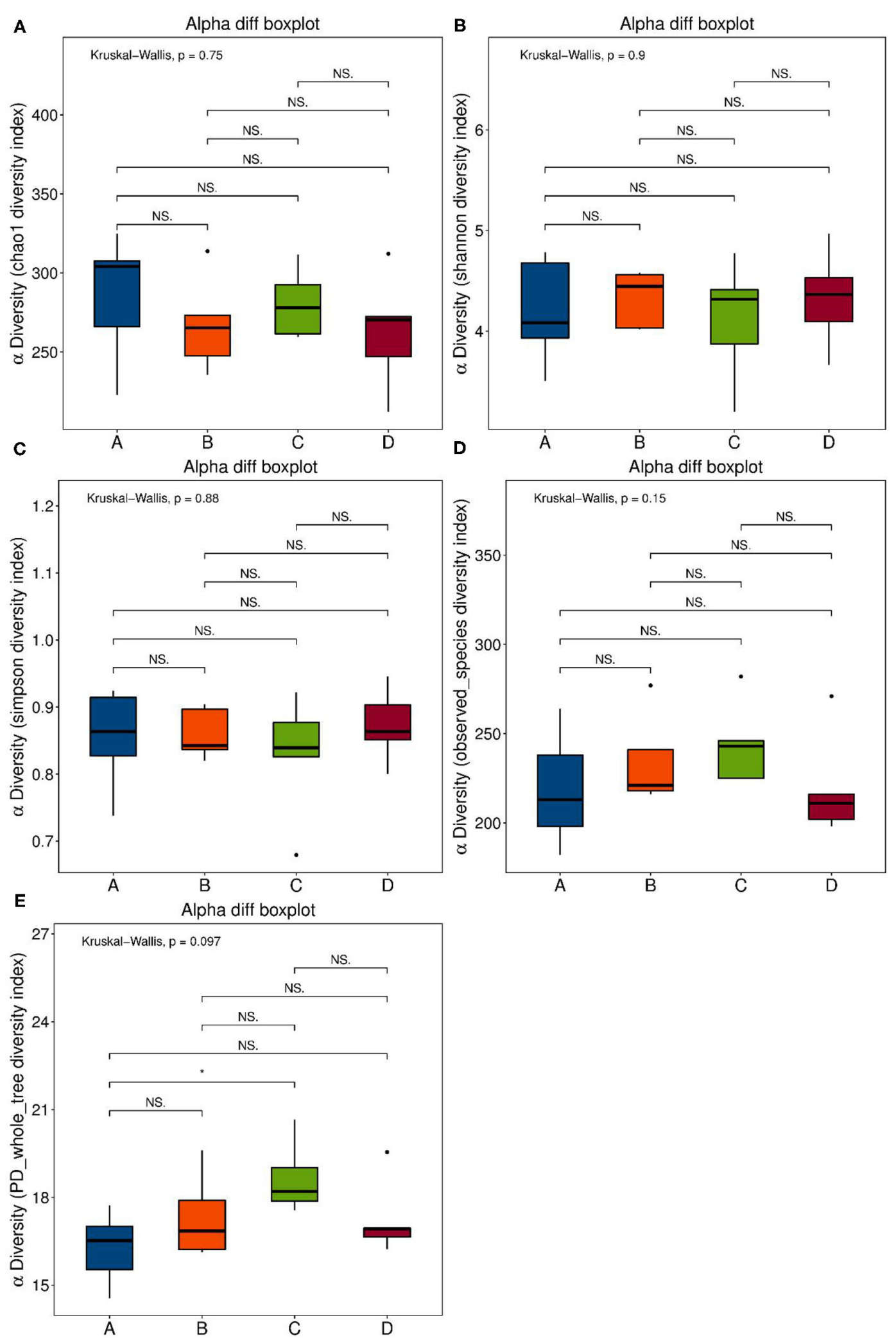

FIGURE 3 | $\alpha$ diversity index. (A) Chao1 index: the differences between the four groups were not statistically significant; (B) Shannon index: the differences between four groups were not statistically significant; (C) Simpson index: the differences between four groups is not statistically significant; (D) Observed-species diversity: the differences between four groups were not statistically significant; (E) PD-whole-tree index: the differences between the four groups were not statistically significant. 


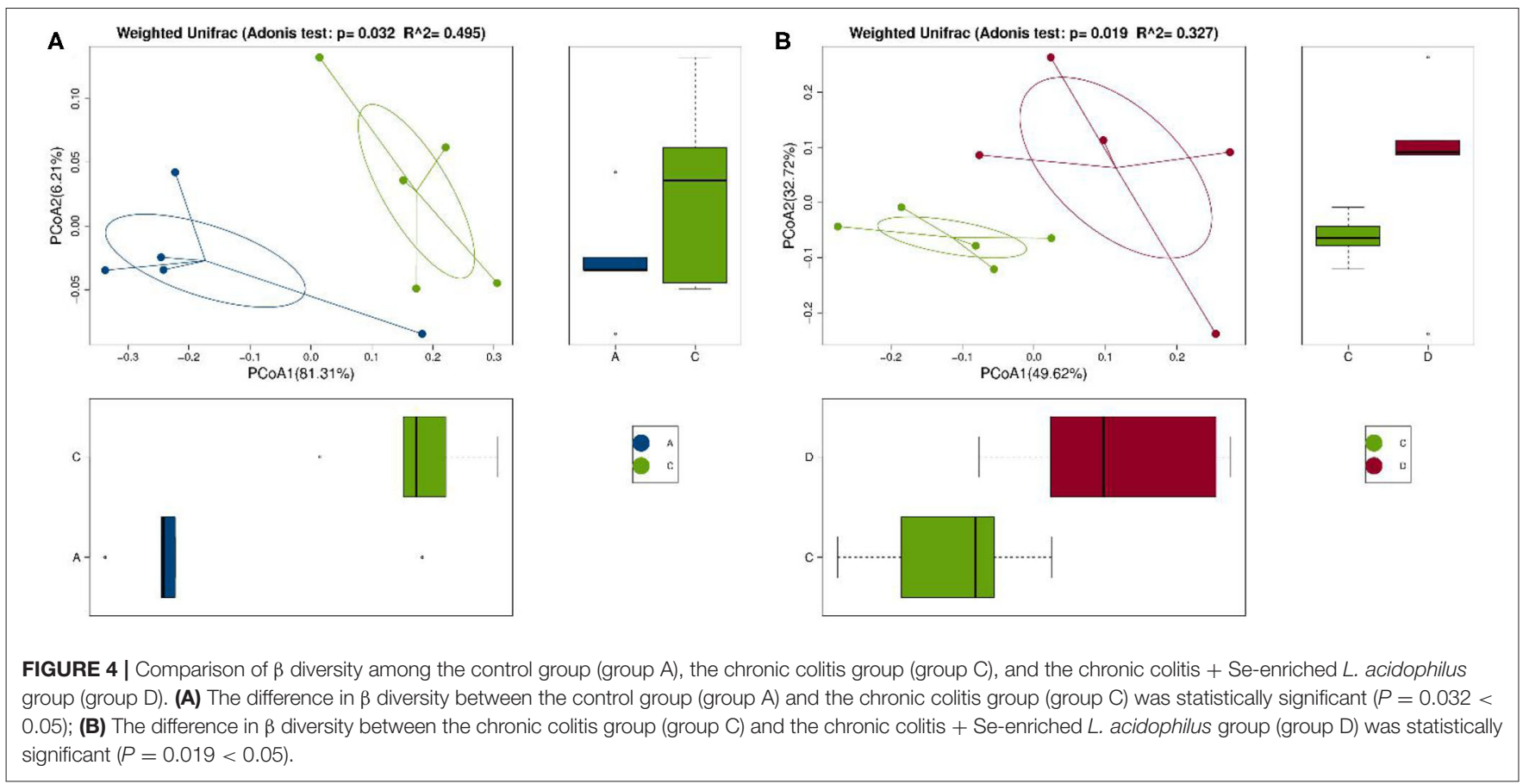

\section{A}

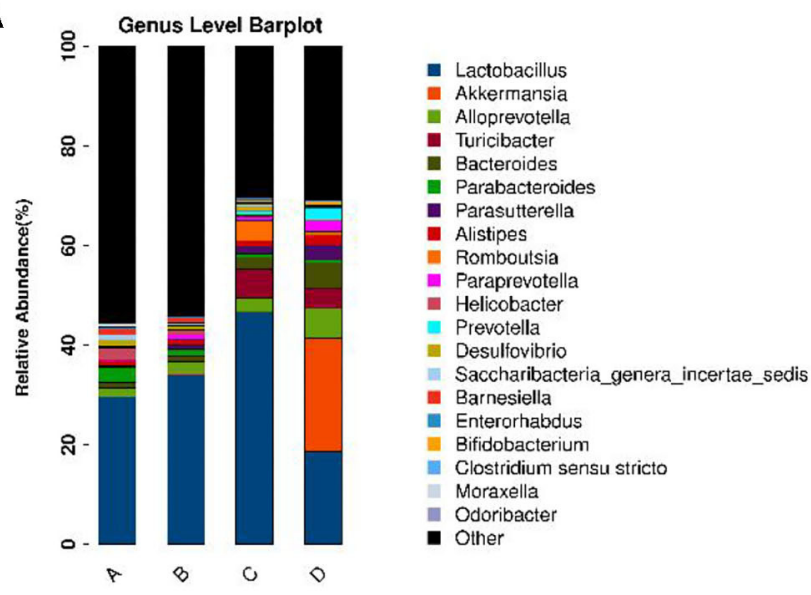

B

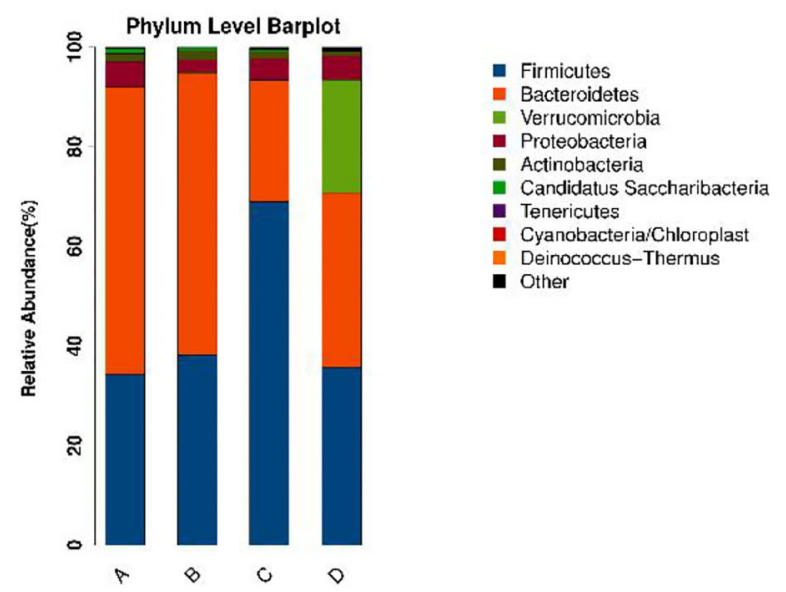

FIGURE 5 | The relative abundance of each microorganism in each group. (A) Genus level; (B) Phylum level.

\section{Correlation Analysis Indicated That Many Species Were Correlated With Cytokines}

In order to identify whether the alteration of microbiota were related to the cytokines, correlation analysis was performed. Akkermansia was positive related to IL-10 $(P<0.05)$. Romboutsia was positive related to TNF- $\alpha$, IL-1 $\beta$, IL-6, IL-23, IL-12p70, IFN- $\gamma$, IL-17A, IL-22, IL-21, and IL-10, as shown in Figure 8.

\section{DISCUSSION}

The beneficial effects of Se and L. acidophilus on IBD have been reported. Se-enriched L. acidophilus may have a certain therapeutic effect on IBD. We found that Se-enriched $L$. acidophilus could alleviate DSS-induced colitis in mice, reduce inflammatory cytokines produced by LPL cells, decrease the relative abundance of Romboutsia and Lactobacillus.

The serum Se concentration of IBD patients decreased (27). A Korean study showed that $30.9 \%$ of IBD patients had Se deficiency (28). Although the details of the relationship between Se and IBD still need further elucidation, animal studies have found that Se can increase CD4 $(+)$ CD25 (+) regulatory T cells and reduce Th1, Th17, and $\gamma \delta$ T cells, thus alleviating DSSinduced colitis (26). Se can transform M1 macrophages into M2 macrophages (29). M1 macrophages promote the development of inflammation, and M2 macrophages have anti-inflammatory effects (30). 


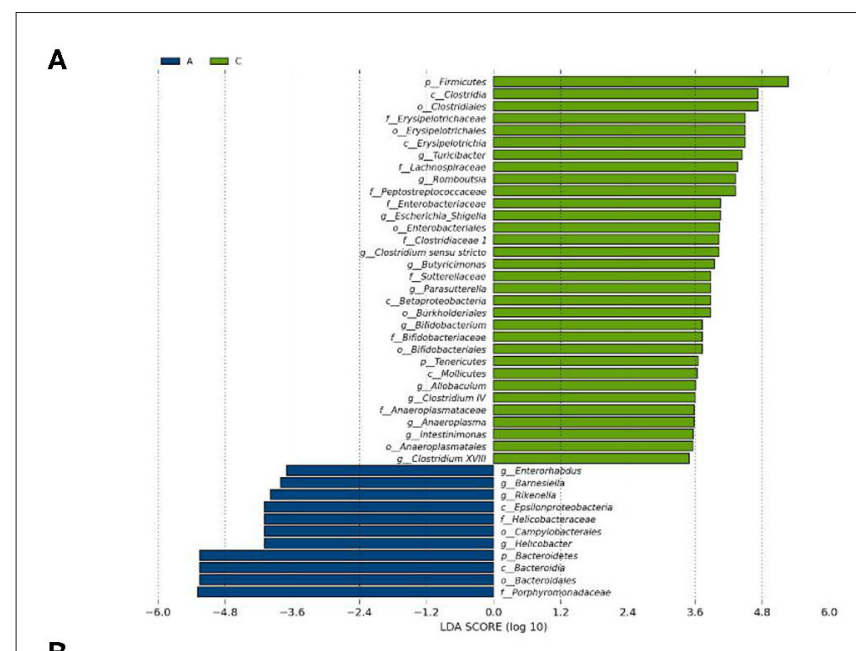

B

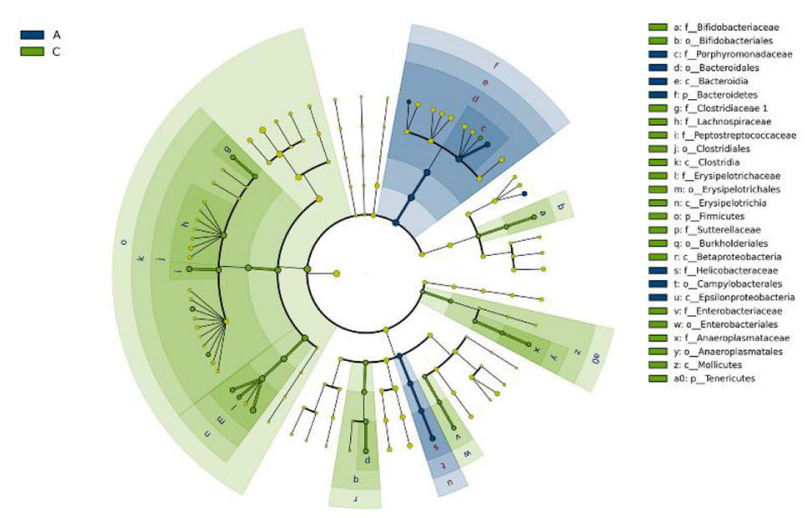

C

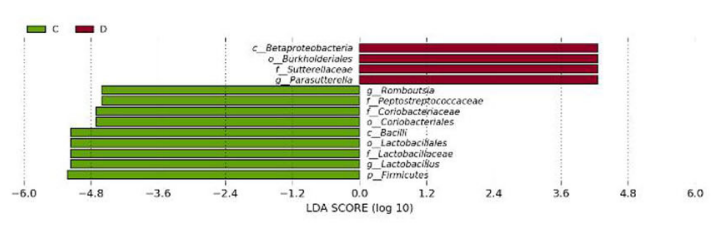

D

붕

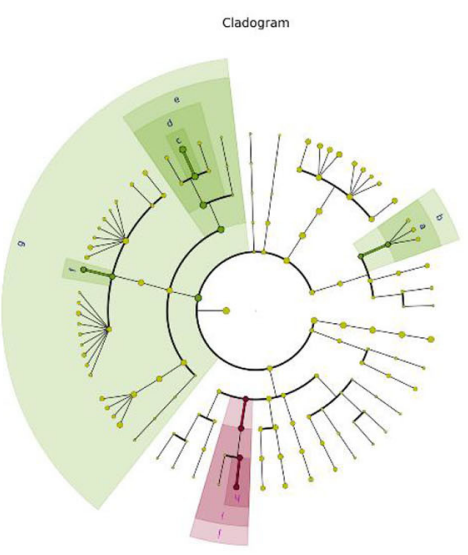

FIGURE 6 | Se-enriched $L$. acidophilus changes the intestinal microbiota. (A) Marker bacteria (LDA score > 2) between the control group (group A) and the

(Continued)
FIGURE 6 | chronic colitis group (group C); (B) A LEfSe cladogram shows the dominant species in the control group (group A) and the chronic colitis group (group C); (C) Marker bacteria (LDA score $>2$ ) between the chronic colitis group (group C) and the chronic colitis + Se-enriched L. acidophilus group (group D); (D) A LEfSe cladogram shows the dominant species of the chronic colitis group (group C) and the chronic colitis + Se-enriched L. acidophilus (group D).

Lactobacillus has long been considered a probiotic. Many studies have reported the therapeutic effects of different Lactobacillus strains on IBD. A randomized clinical trial showed that Lactobacillus reuteri ATCC 55730 enema combined with oral mesalazine can improve the intestinal inflammation of children with mild to moderate active distal ulcerative colitis (31). The Lactobacillus rhamnosus GG strain (LGG) plays a certain role in maintaining the remission stage of ulcerative colitis (32). Animal experiments showed that two Lactobacillus reuteri strains had therapeutic effects on colitis in mice (33). Lactobacillus plantarum 06CC2 has anti-inflammatory effects (34). At the same time, it was found that the intestinal Lactobacillus of mice with DSS-induced colitis decreased (35). This suggested that probiotics belonging to Lactobacillus may be benificial to colitis treatment. However, some studies also found the opposite result: Lactobacillus increased in $\operatorname{IBD}(36,37)$, which is consistent with our result, which may suggest that the intestinal microbiota may have different changes in different stages of IBD. Our study found that Se-enriched L. acidophilus can reduce the relative abundance of Lactobacillus. A previous study also found that Lactobacillus plantarum could reduce the relative abundance of intestinal Lactobacillus in DSS-induced colitis mice (38). These indicate that although the changes in Lactobacillus in IBD need to be further clarified, some probiotics belonging to Lactobacillus may always have certain benefits for the treatment of IBD. Changes in the flora of IBD may be influenced by different situations (gene, diet, immunity, etc.) Therefore, molecular pathological epidemiology (MPE) may be useful for IBD research, and MPE can help doctors better understand the relationship between the flora and the disease. The changes in different flora may be used to distinguish different subtypes of IBD to facilitate more precise and effective treatments.

Se-enriched $L$. acidophilus can reduce the relative abundance of Romboutsia. It has been reported that the relative abundance of Romboutsia in the intestinal microbiota of patients with the autoimmune disease Hashimoto's thyroiditis is increased (39). Our study also found that the relative abundance of Romboutsia in DSS-induced colitis was increased, suggesting that Romboutsia may play a role in promoting autoimmune diseases, which needs further research.

Se-enriched L. acidophilus also increased the abundance of Akkermansia in mice with colitis, although there was no significant difference between the chronic colitis group and the chronic colitis + Se-enriched L. acidophilus group. Our study found that the relative abundance of Akkermansia was similar between the chronic colitis group and the control group. However, increase of the relative abundance of Akkermansia in the intestines of mice with DSS-induced colitis was also reported (40). Akkermansia plays an important role in the intestine. 


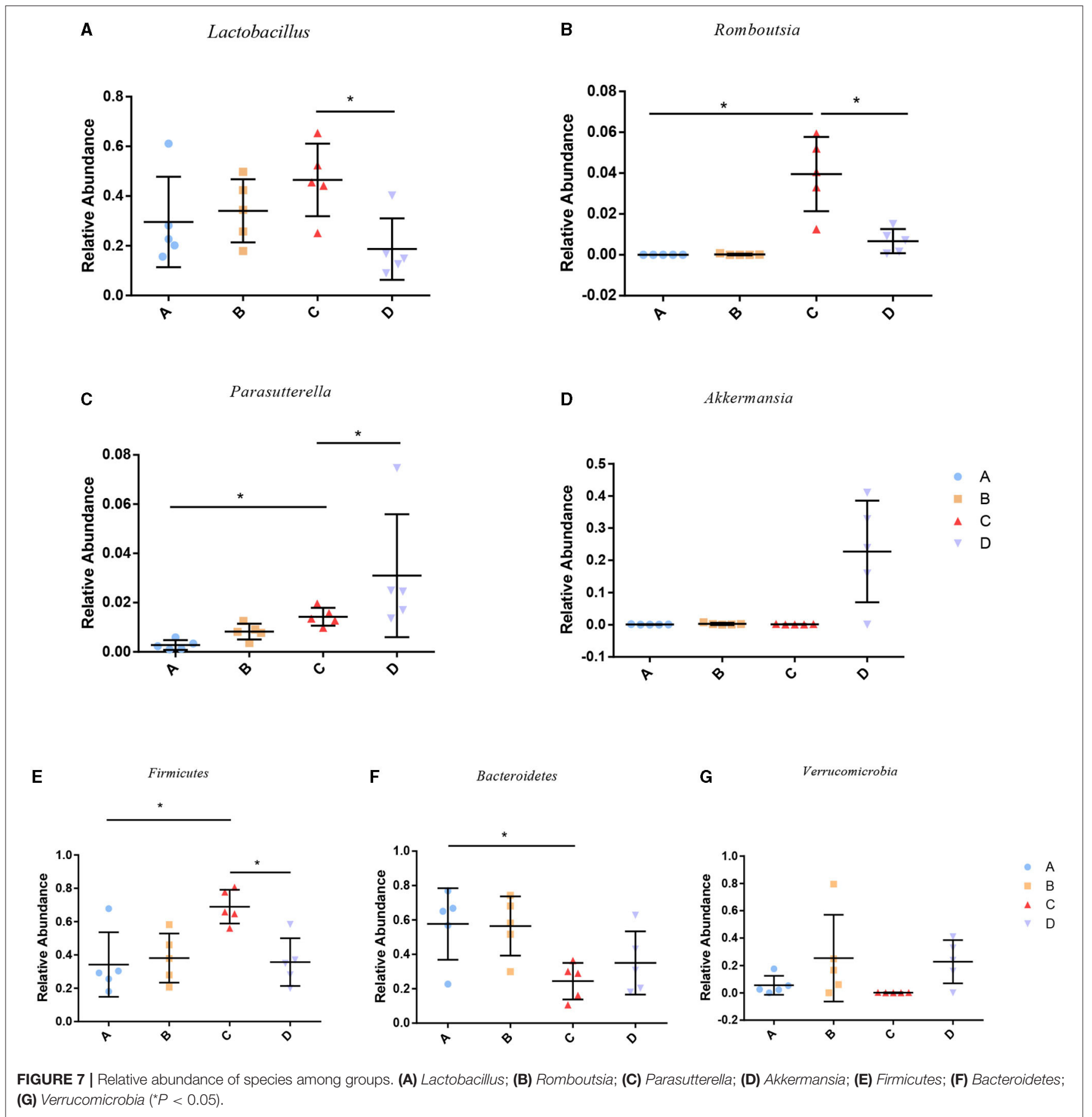

Chlorogenic acid and polyphenol-rich cranberry extract have been reported to alleviate colitis by increasing the abundance of Akkermansia $(41,42)$. Akkermansia muciniphila, a strain of Akkermansia, can maintain intestinal barrier function, reduce the inflammatory response, and alleviate DSS-induced colitis in mice (43). Akkermansia muciniphila extracelluar vesicles help to alleviate the progression of DSS-induced colitis (44). Se also has a certain effect on intestinal microflora. Zhai et al. reported that Se can increase the abundance of Akkermansia in the intestines of mice (45).
Se-enriched L. acidophilus also affects inflammatory cytokines. Cytokines play an important role in the pathogenesis of IBD. The expression levels of TNF- $\alpha$, IL- $1 \beta$, and IFN- $\gamma$ in patients with IBD increased (46). L. acidophilus has inhibitory effects on the proinflammatory factors IL-6, IL-17, IL-1 $\beta$, and TNF$\alpha$ (47). Our study also found that Se-enriched L. acidophilus can inhibit the above proinflammatory cytokines. Another study found that $L$. acidophilus can improve endoplasmic reticulum stress and induce IL-10 production (14). IL-10 is an important anti-inflammatory cytokine in the human 


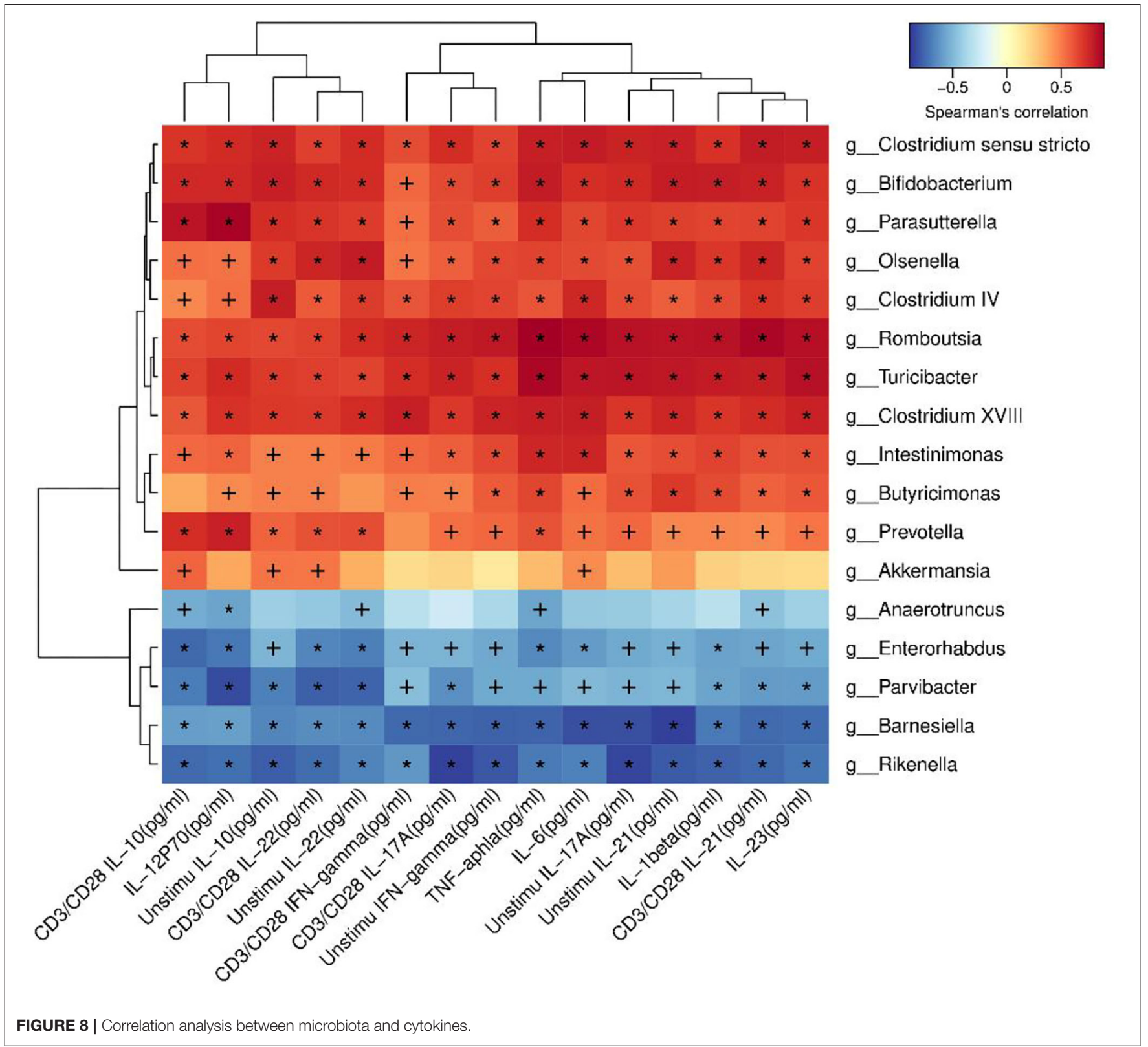

body (48). IL-10 can inhibit the production of IFN- $\gamma$ by CD4+ T cells through dendritic cells (49). IFN- $\gamma$ can increase intestinal vascular permeability and promote the development of intestinal inflammation (50). This mechanism may be related to Akkermansia. It has been reported that Akkermansia is positively correlated with IL-10 (43). We also found that Akkermansia is positively associated with IL-10. IL-21 can induce the initial T cells to differentiate into Th17 cells and produce IL-17 (51). IL17 can promote the production of other inflammatory cytokines (52) and then promote the occurrence and development of inflammation. IL-23 can expand Th17 cells responses (53). IL-1 $\beta$ in synergy with IL- 6 can promote the differentiation of Th17 cells (54). Therefore, inhibition of these inflammatory cytokines helps to alleviate inflammation.
There are still some limitations in this study. The specific mechanisms by which Se-enriched $L$. acidophilus regulates inflammatory cytokines are still unclear. The effect of Se-enriched L. acidophilus metabolites on colitis was not examined in these experiments. Further research should be conducted to clarify this series of problems.

In general, Se-enriched $L$. acidophilus can reduce the production of proinflammatory cytokines in DSS-induced colitis in mice, regulate the intestinal microbiota, and alleviate DSSinduced chronic colitis in mice. Therefore, Se-enriched $L$. acidophilus may have certain therapeutic effects on IBD, especially for patients with reduction of Akkermansia and IL-10, and clinical multicenter studies could be conducted to further study its efficacy in humans. 


\section{DATA AVAILABILITY STATEMENT}

The original contributions presented in the study are included in the article/supplementary material, further inquiries can be directed to the corresponding author/s.

\section{ETHICS STATEMENT}

All specimens from the mice were taken after ethical permission was obtained for participation in the study. The experimental protocols were approved by the Institutional Animal Care and Use Committee of China Medical University.

\section{REFERENCES}

1. Kaser A, Zeissig S, Blumberg RS. Inflammatory bowel disease. Annu Rev Immunol. (2010) 28:573-621. doi: 10.1146/annurev-immunol-030409-101225

2. Sairenji T, Collins KL, Evans DV. An update on inflammatory bowel disease. Prim Care. (2017) 44:673-92. doi: 10.1016/j.pop.2017.07.010

3. Zhang YZ, Li YY. Inflammatory bowel disease: pathogenesis. World J Gastroenterol. (2014) 20:91-9. doi: 10.3748/wjg.v20.i1.91

4. Nishida A, Inoue R, Inatomi O, Bamba S, Naito Y, Andoh A. Gut microbiota in the pathogenesis of inflammatory bowel disease. Clin J Gastroenterol. (2018) 11:1-10. doi: 10.1007/s12328-017-0813-5

5. Ding X, Li Q, Li P, Zhang T, Cui B, Ji G, et al. Long-term safety and efficacy of fecal microbiota transplant in active ulcerative colitis. Drug Saf. (2019) 42:869-80. doi: 10.1007/s40264-019-00809-2

6. Paramsothy S, Kamm MA, Kaakoush NO, Walsh AJ, van den Bogaerde J, Samuel D, et al. Multidonor intensive faecal microbiota transplantation for active ulcerative colitis: a randomised placebo-controlled trial. Lancet. (2017) 389:1218-28. doi: 10.1016/S0140-6736(17)30182-4

7. Costello SP, Hughes PA, Waters O, Bryant RV, Vincent AD, Blatchford P, et al. Effect of fecal microbiota transplantation on 8-week remission in patients with ulcerative colitis: a randomized clinical trial. JAMA. (2019) 321:156-64. doi: 10.1001/jama.2018.20046

8. Miele E, Pascarella F, Giannetti E, Quaglietta L, Baldassano RN, Staiano A. Effect of a probiotic preparation (VSL\#3) on induction and maintenance of remission in children with ulcerative colitis. Am J Gastroenterol. (2009) 104:437-43. doi: 10.1038/ajg.2008.118

9. Mardini HE, Grigorian AY. Probiotic mix VSL\#3 is effective adjunctive therapy for mild to moderately active ulcerative colitis: a meta-analysis. Inflamm Bowel Dis. (2014) 20:1562-7. doi: 10.1097/MIB.0000000000000084

10. Bull M, Plummer S, Marchesi J, Mahenthiralingam E. The life history of Lactobacillus acidophilus as a probiotic: a tale of revisionary taxonomy, misidentification and commercial success. FEMS Microbiol Lett. (2013) 349:77-87. doi: 10.1111/1574-6968.12293

11. Lee SH, Kwon JY, Jhun J, Jung K, Park SH, Yang CW, et al. Lactobacillus acidophilus ameliorates pain and cartilage degradation in experimental osteoarthritis. Immunol Lett. (2018) 203:6-14. doi: 10.1016/j.imlet.2018. 07.003

12. Yan F, Li N, Shi J, Li H, Yue Y, Jiao W, et al. Lactobacillus acidophilus alleviates type 2 diabetes by regulating hepatic glucose, lipid metabolism and gut microbiota in mice. Food Funct. (2019) 10:5804-15. doi: 10.1039/C9FO01062A

13. Park SS, Lee YJ, Song S, Kim B, Kang H, Oh S, et al. Lactobacillus acidophilus NS1 attenuates diet-induced obesity and fatty liver. J Endocrinol. (2018) 237:87-100. doi: 10.1530/JOE-17-0592

14. Kim DH, Kim S, Lee JH, Kim JH, Che X, Ma HW, et al. Lactobacillus acidophilus suppresses intestinal inflammation by inhibiting endoplasmic reticulum stress. J Gastroenterol Hepatol. (2019) 34:178-85. doi: $10.1111 /$ jgh. 14362

\section{AUTHOR CONTRIBUTIONS}

DP performed animal and molecular biology experiments. ZW and LS analyzed and interpreted the data and wrote the manuscript. MJ conceived and designed the study. All authors approved the final manuscript.

\section{FUNDING}

This work was Supported by the innovative talent support program of the Institute of Higher Learning of Liao Ning Province (No. 2018-478) and the 2020 Shenyang Science and Technology Plan (Second Batch) (20-205-4-094).

15. Wang MX, Lin L, Chen YD, Zhong YP, Lin YX, Li P, et al. Evodiamine has therapeutic efficacy in ulcerative colitis by increasing Lactobacillus acidophilus levels and acetate production. Pharmacol Res. (2020) 159:104978. doi: 10.1016/j.phrs.2020.104978

16. Avery JC, Hoffmann PR. Selenium, selenoproteins, and immunity. Nutrients. (2018) 10:1203. doi: 10.3390/nu10091203

17. Fairweather-Tait SJ, Bao Y, Broadley MR, Collings R, Ford D, Hesketh JE, et al. Selenium in human health and disease. Antioxid Redox Signal. (2011) 14:1337-83. doi: 10.1089/ars.2010.3275

18. Andoh A, Hirashima M, Maeda H, Hata K, Inatomi O, Tsujikawa T, et al. Serum selenoprotein-P levels in patients with inflammatory bowel disease. Nutrition. (2005) 21:574-9. doi: 10.1016/j.nut.2004.08.025

19. Reimund JM, Hirth C, Koehl C, Baumann R, Duclos B. Antioxidant and immune status in active Crohn's disease. A possible relationship. Clin Nutr. (2000) 19:43-8. doi: 10.1054/clnu.1999.0073

20. Geerling BJ, Badart-Smook A, Stockbrügger RW, Brummer RJ. Comprehensive nutritional status in recently diagnosed patients with inflammatory bowel disease compared with population controls. Eur J Clin Nutr. (2000) 54:514-21. doi: 10.1038/sj.ejcn.1601049

21. Sang L, Chang B, Zhu J, Yang F, Li Y, Jiang X, et al. Dextran sulfate sodiuminduced acute experimental colitis in C57BL/6 mice is mitigated by selenium. Int Immunopharmacol. (2016) 39:359-68. doi: 10.1016/j.intimp.2016.07.034

22. Jin W, Yoon C, Johnston TV, Ku S, Ji GE. Production of selenomethionineenriched Bifidobacterium bifidum BGN4 via sodium selenite biocatalysis. molecules. (2018) 23:2860. doi: 10.3390/molecules23112860

23. Chen L, Pan DD, Zhou J, Jiang YZ. Protective effect of seleniumenriched Lactobacillus on CCl4-induced liver injury in mice and its possible mechanisms. World J Gastroenterol. (2005) 11:5795-800. doi: 10.3748/wjg.v11.i37.5795

24. Liu Y, Liu Q, Ye G, Khan A, Liu J, Gan F, et al. Protective effects of Seleniumenriched probiotics on carbon tetrachloride-induced liver fibrosis in rats. $J$ Agric Food Chem. (2015) 63:242-9. doi: 10.1021/jf5039184

25. Malyar RM, Naseri E, Li H, Ali I, Farid RA, Liu D, et al. Hepatoprotective effects of selenium-enriched probiotics supplementation on heat-stressed wistar rat through anti-inflammatory and antioxidant effects. Biol Trace Elem Res. (2020) 199:3445-56. doi: 10.1007/s12011-020-02475-3

26. Sang LX, Chang B, Zhu JF, Yang FL, Li Y, Jiang XF, et al. Sodium selenite ameliorates dextran sulfate sodium-induced chronic colitis in mice by decreasing Th1, Th17, and $\gamma \delta \mathrm{T}$ and increasing CD4(+)CD25(+) regulatory T-cell responses. World J Gastroenterol. (2017) 23:3850-63. doi: 10.3748/wjg.v23.i21.3850

27. Castro Aguilar-Tablada T, Navarro-Alarcón M, Quesada Granados J, Samaniego Sánchez C, Rufián-Henares JÁ, Nogueras-Lopez F. Ulcerative colitis and crohn's disease are associated with decreased serum selenium concentrations and increased cardiovascular risk. Nutrients. (2016) 8:780. doi: $10.3390 /$ nu8120780

28. Han YM, Yoon H, Lim S, Sung MK, Shin CM, Park YS, et al. Risk factors for vitamin D, zinc, and selenium deficiencies in Korean 
patients with inflammatory bowel disease. Gut Liver. (2017) 11:363-9. doi: 10.5009/gnl16333

29. Nelson SM, Lei X, Prabhu KS. Selenium levels affect the IL-4-induced expression of alternative activation markers in murine macrophages. J Nutr. (2011) 141:1754-61. doi: 10.3945/jn.111.141176

30. Lawrence T, Natoli G. Transcriptional regulation of macrophage polarization: enabling diversity with identity. Nat Rev Immunol. (2011) 11:750-61. doi: $10.1038 /$ nri3088

31. Oliva S, Di Nardo G, Ferrari F, Mallardo S, Rossi P, Patrizi G, et al. Randomised clinical trial: the effectiveness of Lactobacillus reuteri ATCC 55730 rectal enema in children with active distal ulcerative colitis. Aliment Pharmacol Ther. (2012) 35:327-34. doi: 10.1111/j.1365-2036.2011.04939.x

32. Zocco MA, dal Verme LZ, Cremonini F, Piscaglia AC, Nista EC, Candelli M, et al. Efficacy of Lactobacillus GG in maintaining remission of ulcerative colitis. Aliment Pharmacol Ther. (2006) 23:1567-74. doi: 10.1111/j.1365-2036.2006.02927.x

33. Ahl D, Liu H, Schreiber O, Roos S, Phillipson M, Holm L. Lactobacillus reuteri increases mucus thickness and ameliorates dextran sulphate sodiuminduced colitis in mice. Acta Physiol. (2016) 217:300-10. doi: 10.1111/apha. 12695

34. Tanaka A, Kanmura S, Morinaga Y, Kawabata K, Arima S, Sasaki F, et al. Oral administration of Lactobacillus plantarum 06CC2 prevents experimental colitis in mice via an anti-inflammatory response. Mol Med Rep. (2020) 21:1181-91. doi: $10.3892 / \mathrm{mmr} .2020 .10925$

35. Wang J, Zhang C, Guo C, Li X. Chitosan ameliorates DSS-induced ulcerative colitis mice by enhancing intestinal barrier function and improving microflora. Int J Mol Sci. (2019) 20:5751. doi: 10.3390/ijms20225751

36. Wang W, Chen L, Zhou R, Wang X, Song L, Huang S, et al. Increased proportions of Bifidobacterium and the Lactobacillus group and loss of butyrate-producing bacteria in inflammatory bowel disease. J Clin Microbiol. (2014) 52:398-406. doi: 10.1128/JCM.01500-13

37. Takaishi H, Matsuki T, Nakazawa A, Takada T, Kado S, Asahara T, et al. Imbalance in intestinal microflora constitution could be involved in the pathogenesis of inflammatory bowel disease. Int J Med Microbiol. (2008) 298:463-72. doi: 10.1016/j.jimm.2007.07.016

38. Zhang F, Li Y, Wang X, Wang S, Bi D. The impact of Lactobacillus plantarum on the gut microbiota of mice with DSS-induced colitis. Biomed Res Int. (2019) 2019:3921315. doi: 10.1155/2019/3921315

39. Zhao F, Feng J, Li J, Zhao L, Liu Y, Chen H, et al. Alterations of the gut microbiota in Hashimoto's thyroiditis patients. Thyroid. (2018) 28:175-86. doi: 10.1089/thy.2017.0395

40. Berry D, Schwab C, Milinovich G, Reichert J, Ben Mahfoudh K, Decker T, et al. Phylotype-level 16S rRNA analysis reveals new bacterial indicators of health state in acute murine colitis. ISME J. (2012) 6:2091-106. doi: 10.1038/ismej.2012.39

41. Zhang Z, Wu X, Cao S, Cromie M, Shen Y, Feng Y, et al. Chlorogenic acid ameliorates experimental colitis by promoting growth of akkermansia in mice. Nutrients. (2017) 9:677. doi: 10.3390/nu9070677

42. Anhê FF, Roy D, Pilon G, Dudonné S, Matamoros S, Varin TV, et al. A polyphenol-rich cranberry extract protects from diet-induced obesity, insulin resistance and intestinal inflammation in association with increased Akkermansia spp. population in the gut microbiota of mice. Gut. (2015) 64:872-83. doi: 10.1136/gutjnl-2014-307142

43. Bian $\mathrm{X}, \mathrm{Wu} \mathrm{W}$, Yang $\mathrm{L}, \mathrm{Lv} \mathrm{L}$, Wang $\mathrm{Q}, \mathrm{Li}$ Y, et al. Administration of Akkermansia muciniphila ameliorates dextran sulfate sodiuminduced ulcerative colitis in mice. Front Microbiol. (2019) 10:2259. doi: $10.3389 /$ fmicb.2019.02259
44. Kang CS, Ban M, Choi EJ, Moon HG, Jeon JS, Kim DK, et al. Extracellular vesicles derived from gut microbiota, especially Akkermansia muciniphila, protect the progression of dextran sulfate sodium-induced colitis. PLOS ONE. (2013) 8:e76520. doi: 10.1371/journal.pone.0076520

45. Zhai Q, Cen S, Li P, Tian F, Zhao J, Zhang H, et al. Effects of dietary selenium supplementation on intestinal barrier and immune responses are associated with its modulation of gut microbiota. J Environ Sci Technol Lett. (2018). doi: 10.1021/acs.estlett.8b00563

46. Singh UP, Singh NP, Murphy EA, Price RL, Fayad R, Nagarkatti M, et al. Chemokine and cytokine levels in inflammatory bowel disease patients. Cytokine. (2016) 77:44-9. doi: 10.1016/j.cyto.2015.10.008

47. Park JS, Choi JW, Jhun J, Kwon JY, Lee BI, Yang CW, et al. Lactobacillus acidophilus improves intestinal inflammation in an acute colitis mouse model by regulation of Th17 and treg cell balance and fibrosis development. J Med Food. (2018) 21:215-24. doi: 10.1089/jmf.2017.3990

48. Ouyang W, Rutz S, Crellin NK, Valdez PA, Hymowitz SG. Regulation and functions of the IL-10 family of cytokines in inflammation and disease. Annu Rev Immunol. (2011) 29:71-109. doi: 10.1146/annurev-immunol-031210-101312

49. Veenbergen S, Li P, Raatgeep HC, Lindenbergh-Kortleve DJ, SimonsOosterhuis Y, Farrel A, et al. IL-10 signaling in dendritic cells controls IL- $1 \beta$-mediated IFN $\gamma$ secretion by human $\mathrm{CD} 4+\mathrm{T}$ cells: relevance to inflammatory bowel disease. Mucosal Immunol. (2019) 12:1201-11. doi: 10.1038/s41385-019-0194-9

50. Langer V, Vivi E, Regensburger D, Winkler TH, Waldner MJ, Rath T, et al. IFN- $\gamma$ drives inflammatory bowel disease pathogenesis through VE-cadherindirected vascular barrier disruption. J Clin Invest. (2019) 129:4691-707. doi: 10.1172/JCI124884

51. Fina D, Sarra M, Fantini MC, Rizzo A, Caruso R, Caprioli F, et al. Regulation of gut inflammation and th17 cell response by interleukin-21. Gastroenterology. (2008) 134:1038-48. doi: 10.1053/j.gastro.2008.01.041

52. Neurath MF. Cytokines in inflammatory bowel disease. Nat Rev Immunol. (2014) 14:329-42. doi: 10.1038/nri3661

53. Sarra M, Pallone F, Macdonald TT, Monteleone G. IL-23/IL-17 axis in IBD. Inflamm Bowel Dis. (2010) 16:1808-13. doi: 10.1002/ibd.21248

54. Mao L, Kitani A, Strober W, Fuss IJ. The role of NLRP3 and IL-1 $\beta$ in the pathogenesis of inflammatory bowel disease. Front Immunol. (2018) 9:2566. doi: 10.3389/fimmu.2018.02566

Conflict of Interest: The authors declare that the research was conducted in the absence of any commercial or financial relationships that could be construed as a potential conflict of interest.

Publisher's Note: All claims expressed in this article are solely those of the authors and do not necessarily represent those of their affiliated organizations, or those of the publisher, the editors and the reviewers. Any product that may be evaluated in this article, or claim that may be made by its manufacturer, is not guaranteed or endorsed by the publisher.

Copyright $\odot 2021 \mathrm{Wu}$, Pan, Jiang, Sang and Chang. This is an open-access article distributed under the terms of the Creative Commons Attribution License (CC BY). The use, distribution or reproduction in other forums is permitted, provided the original author(s) and the copyright owner(s) are credited and that the original publication in this journal is cited, in accordance with accepted academic practice. No use, distribution or reproduction is permitted which does not comply with these terms. 